$\begin{array}{llllr}\text { Silver succinate } & \mathrm{Ag}_{2} \mathrm{C}_{4} \mathrm{H}_{4} \mathrm{O}_{4} & \text { Substance taken } & 0.1124 \mathrm{~g} . \\ & \mathrm{AgCl} & 0.0969 \mathrm{~g} . & \mathrm{Ag} & 0.0729 \mathrm{gg} \\ & \mathrm{Ag} & & \text { Found } & 64.89 \% \\ & & & \text { Calc. } & 65.02 \%\end{array}$

From the culture of calcium acetate medium at $25-30^{\circ} \mathrm{C}$ for 60 days, succinic acid identical with the above description was obtained.

\title{
A NEW BUTYL AND ISOPROPYL ALCOHOLS FERMENTATION.
}

\author{
By Kisaku Morikawa. \\ (Received Feb. 8th., 1927)
}

The production of higher alchols for use as solvents and in many technical developments has made great headway in the past ten years. The work of Speakman in Canada, Northrup and Fred in America and of a number of investigators in Europe has been especially worthy of mention.

It is the belief of the author of this investigation that many fermentations as yet undesscribed may have technical significance and find use in industry. The present research represents one study of this character. In this I have dealt with the fermentations of Kojimash, much used in my country (Japan) and which contains large amounts of fermentable sugars.

While making some experiments in this field, I isolated certain organisms which develop and produce fermentation under aerobic conditions with the formation of what appeared to be butyl and isopropyl alcohols as the chief products of the fermentation. Continued work with pure cultures led to this investigation, in which I have described the morphology and physiology of a new organism, and have recorded the results of many studies of its fermentation reactions on various linds of subtrates.

Media-For these studies the standard media of the American B icteriological Society were usually employed, in addition to which I have used Koji broth, Koji-agar, malt extract broth and its agar, 5\% corn meal mash with calcium carbonate and other kinds of sugar broths.

Studies on the Microorganisms.

Morphology-Vegetative cells appear in single rods, in pairs or in chains, having rounded ends, and enclosing granules in their cell especially in old 
stages. Size of rods shows somewhat wide variability, 2 to $5 \mu$, majority about $3 \mu$ in length, 0.7 to $1.5 \mu$, majority ahout $1.0 \mu$ in width. Sporangia appear slightly swollen and with an ellipsoidal or clavated structure enclosing a spore in each cell, 2 to $5 \mu$, majority $3 \mu$ in length, 1.25 to $1.5 \mu$, majority $1.25 \mu$ in width. Spores are thick walled cells located at center or somewhat eccentric and having ellipsoidal structure, 1.8 to $2.5 \mu$, majority $2 \mu$ in length, 0.6 to $1.25 \mu$, majority $1.0 \mu$ in width. In the vegetative stage motility is very active in broth or agar cultures especially at a young stage having flagella. In old cultures it shows quite a variability of forms, swollen or somewhat clavated, and there appear difficulty stainable cells for most dyes especially methylene blue.

\section{Cultural Characteristics.}

Agar stroke : With plain, Koji or malt agar, it shows good growth having echinulate, slightly raised elevation, glistening luster, smooth or little contoured surface, opaqueness, and butyrous consistency.

Gelatin stab : Liquefied medium comparatively rapidly showing good growth especially at the top.

Potato : Shows good growth with dirty white luster. Especially in the older stage it has a tendency to develop brownish colour and appears butyrous consistency of colony.

Nutrient broth : Produce good growth, strong clouding and abundant compact sediment.

Milk : Forms gas and acid, coagulating casein, afterwards digests protein very weakly. In litmus milk nearly the same character is observed without any injurious effects of litmus added. The colour of litmus is reduced gradually within a week.

Agar plate: In plain agar medium it grows rapidly appearing as circular colonies margined irregularly, smooth or rough surface, flat elevation, and shows finely curled internal structure.

Starch digestion : On starch agar plate culture no marked digestion is recognized, but with thick corn meal mush it reduces the viscosity forming gas and watery liquid.

Gas production : With arabinose, xylose, rhamnose, glucose, fructose, galactose, lactose, raffinose, inuline, mannite, dulcite, adonite, glycerin, and inosite broths, little or much gas is produced depending on the kind of substances.

Indol production : With peptone water or nutrient broth no indol is formed.

Hydrogen sulphide production: With various kinds of sodium acetate 
media no hydrogen sulphide is recognized.

Nitrate reduction and ammonium production : With potassium nitrate broth or agar, nitrate is reduced into nitrite, besides ammonia is formed.

Temperature relations : Optimum $30^{\circ}-40^{\circ} \mathrm{C}$., minimum $10^{\circ} \mathrm{C}$, maximum $50^{\circ} \mathrm{C}$.

Relation to oxygen : Good growth in aerobic or partial anaerobic condition.

Relation to reaction of medium : Optimum $\mathrm{pH} 70$. to $\mathrm{pH} 60$.

Endo's agar stab : Bright red colouration, moderate growth along the needle track; slight gas is formed.

Methyl red test : Strikingly positive reaction is exposed.

The products of fermentation : Raw starch is not affected by this organism, but sugars are readily fermented yielding normal butyl and isopropyl alcohols as the chief end products. Small quantities of acetic and butyric acid are produced. The gas evolv.d during fermentation consists of $\mathrm{H}$ and $\mathrm{CO}_{2}$, the hydrogen greatly exceeding the $\mathrm{CO}_{2}$ in volume, and the ratio varying at different periods of the fermentation. Under semianaerobic conditions, that is, with reduced oxygen tension, small quantities of acetone are found in the distillation products.

Careful comparative studies have demonstrated that the organism is unlike any other yielding similar products which have previously been described. Most of the organisms which yield alcohols, acetone and fatty acids which have been studied up to this time may be divided into two groups.

(1) Those which produce higher alcohols and acetone under anaerobic conditions.

(2) Those which yield ethyl alcohol and acetone under aerobic or facultatively anaerobic conditions.

I have found no ethyl alcohol, under any of the conditions studied.

In view of these differences in morphology and physiological behavior, I believe this to be a new species and have given it the provisional name, Bacillus Technicus, n. sp. Prescott and Morikawa.

Further Studes on BIochemistey of tile OrGianism.

To obtain more detailed information of physiological features, further experiments such as sugar reactions, change of gas volume which may be produced, acidity changes during incubation, and injurious effects of products on bacterial growth have been studied. All sugars tested have been consumed causing much or little gas evolution and increasing acidity. Of the pentoses. thamnose gave little gas and allaline reaction after seven days 
fermentation, while arabinose and xylose were strongly acid and gave abundant gas formation. All the monosaccharides and disaccharides gave high acidity and much gas. The polyatomic alcohols adonite, dulcite and mannite and glycerin gave little gas. With adonite and dulcite the final reaction was alkaline, while with glycerin and mannite it was slightly acid. The alkaline reaction with adonite and dulcite is apparently the result of action on the nitrogenous constituents of the medium by which it is broken down to amines and ammonia.

Studies on the occurrence of aldehydes as an intermediate product were positive to Schiff's test in all cases. It may be supposed that the sugars are converted into aldehydes, which is instantly transformed by reduction into alcohols or by oxidation to corresponing acids.

In the early hours of the fermentation vigorous gas production and the high percentage of hydrogen in the gas evolved may be noted. This is probably due to the greater solubility of the carbon dioxide in the medium. At the intermediate period of duration the low percentage may appear, which is owing to the saturation of the solution by carbon dioxide.

An approximate ratio of hydrogen and carbon dioxide resulted 4.5 to 6.4 showing somewhat wide variation which may depend on the condition of media and the stages of fermentation tested. As to acidity change during fermentation in my process, the hydrogen ion change may show somewhat unclulatory changes, that is, a rise and fall of concentration, while the titrable acidity change appears to be quite identical with that noted by others with B. granulobacter pectinovorum. In my investigations it was found that the titrable acidity may increase comparatively quickly, depending on the conditions, until it reaches the maximum and then it may decrease gradually to the minimum. There is not an acute increase and decrcase of the acidity as in the case of B. granulobacter pectinovorum with corn mash.

This organism appears to be very sensitive to conditions in the medium. If there are some injurious substances in a prepared mash, the bacterial growth may be prevented. For example, with too high acidity, excessive sugar concentration or presence of a certain amount of alcohols, the bacterial growth may not appear. Although the organism here described is very sensitive to the hydrogen ion concentration at the starting point of fermentation, it may be active until about pH 4.6 with Koji mash. On the other hand, in dextrose broth this organism will grow very poorly, if at all, in $\mathrm{pH}$ 5.0 or a stronger acidity. At the end of fermentation, normally a fermented mash will show pH 4.4 to 4.2 , or somewhat low hydrogen ion concentration, and titrable acidity 3.0 c.c. of $\mathrm{n} / 10$ sodium hydroxide solution per 10c.c. of fermented mash. In both cases, the results may be affected by the presence 
of neutralizing agents. Among organic acids, which were supposed to be injurious to bacterial growth, lactic acid displayed a strongly inhibitive action, and butyric and acetic acids had weaker action in preventing growth or fermentation. This may be explainable, since the latter two acids have smaller ionization constants than the former. By an addition of about one percent of butyl alcohol before inoculation to dextrose broth, no fermentation will occur or it may be retarded, but ethyl and isopropyl alcohols may not be so harmful as butyl alcohol. The effect of their own metabolic products which are obtained from old cultures by filtering through a Berkefeld filter may be harmless in the Koji mash within certain limits.

A fermentation process has been developed, which is quite different from others now in use in which starch is converted into sugars by enzyme action of molded rice. The sugars thus produced are converted into butyl and isopropyl alcohols by the $\mathbf{B}$. technicus under aerobic conditions. The consumption of sugar is most rapid in the earlier stage of fermentation, and afterwards it may show a slow decrease of the sugar content. These changes occurring somewhat in correlation with the gas production and acidity of the mash. On the contrary, the formation of alcohols may appear especially in the final stage of a fermentation. Although much unutilized sugar may remain in the fermented mash at the end of a fermentation, a good yield may be obtained in a fairly concentrated sugar mash, $12 \%$ of sugar as glucuse in a mash being most favorable.

From the many experimental results it was found that 100 grams of sugar as glucose produced 30 grams of alcohol, and assuming that two mols of butyl alcohol and one mol of isopropyl alcohol may be formed from three mols of glucose, but sometimes the ratio of butyl and isopropyl alcohols showed $3: 1$. The yield of alcohols presumably is mostly affected by the concentration of sugar, total volume and hydrogen ion concentration of the mash. Normally 20c.c. or somewhat more alcohols may be obtained from one liter of mash. A specially high percentage yield cannot be expected since the solubility of butyl alcohol is limited, and because of the inhibiting nature of alcohols for the organisms. The determination of products such as alcohols and acids was made by testing their physical and chemical properties. Especially the alcohols were confirmed by ester formation with 3.5 dinitrobenzoic acid, and furthermore, isopropyl alcohol was tested by oxidation and then condensation with benzaldehyde. Acids were identified as toluides with paratoluidine or as paranitrobenzyl esters. 